\title{
Silent brain infarction: a quiet predictor of future stroke
}

\author{
Oh Young Bang ${ }^{1,2}$ \\ ${ }^{1}$ Department of Neurology, Samsung Medical Center, Sungkyunkwan University School of Medicine, Seoul, Korea \\ ${ }^{2}$ Translational and Stem Cell Research Laboratory on Stroke, Samsung Medical Center, Seoul, Korea
}

Received: July 16, 2018

Revised: July 27, 2018

Accepted: August 1, 2018

Corresponding author:

Oh Young Bang

Department of Neurology,

Samsung Medical Center,

Sungkyunkwan University

School of Medicine, 81 Irwon-

ro, Gangnam-gu, Seoul 06351,

Korea

Tel: +82-2-3410-3599

E-mail:

ohyoung.bang@samsung.com

\section{ABSTRACT}

A silent brain infarct (SBI) is defined as imaging or neuropathological evidence of brain infarction without a history of acute neurological dysfunction attributable to the lesion. The number of patients with SBIs is estimated as several-fold higher than the number with clinical stroke. In addition, SBIs have important clinical implications. The presence of SBIs more than doubles the risk of subsequent stroke and dementia. Although most SBIs are lacunes, for which hypertensive small vessel disease is thought to be the main cause, some of them could be embolic in origin. The pathological mechanisms of SBIs and most effective strategies for prevention of future stroke may differ depending on the cause of the SBI. The literature reviewed and cases presented herein underscore the need for application of appropriate workups and therapeutic strategies in patients with SBIs. In this review, the definition, causes, and clinical impact of SBIs are discussed, together with the questions that remain open and recent advances (e.g., machine learning techniques) in the study of SBIs.

Keywords: Brain ischemia; Etiology; Silent brain infarction; Stroke; Stroke subtype
This is an Open Access article distributed under the terms of the Creative Commons Attribution Non-Commercial License (http:// creativecommons.org/licenses/ by-nc/4.0/).

\section{INTRODUCTION: DO I HAVE A SILENT STROKE?}

Nowadays, the role of imaging in stroke diagnosis is increasing and magnetic resonance imaging (MRI) is becoming more widely available. Three healthy-appearing women in their 60 s visited an outpatient clinic with brain MRI scans taken as part of a health checkup. All of them were concerned about abnormal findings on their MRIs, and asked whether they had brain infarcts. The first woman (case A), with no vascular risk factors such as hypertension or diabetes, had a fluid-attenuated inversion recovery (FLAIR) scan showing small spots in the white matter. However, no signal changes were observed in T1- and T2-weighted images, and she was reassured that the MRI findings were unidentified bright objects (UBOs), indicating nonspecific white matter changes (Fig. 1A). The second woman (case B), who had hypertension, showed leukoaraiosis in the periventricular white matter and deep brain areas, which are often seen in the normal elderly but are also seen in association with vascular risk factors or in the context of cognitive impairment (Fig. 1B) [1]. The third woman (case C) showed silent brain infarctions (SBIs) in deep brain areas on FLAIR (Fig. 1C). Unlike UBO, these SBIs appeared hyperintense on 
T2- and hypointense on T1-weighted images. The magnetic resonance (MR) angiography showed normal findings, suggesting that $\mathrm{SBI}$ was caused by microangiopathy.
Patients may have silent but acute brain infarcts that can be embolic in origin. A 62-year-old apparently healthy woman (case D) with a history of migraine headache underwent
Case A

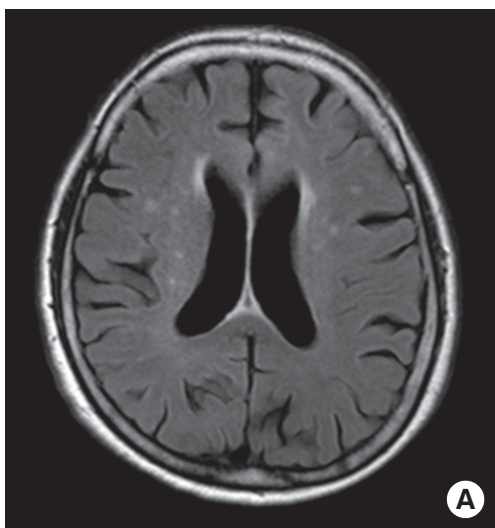

Case B

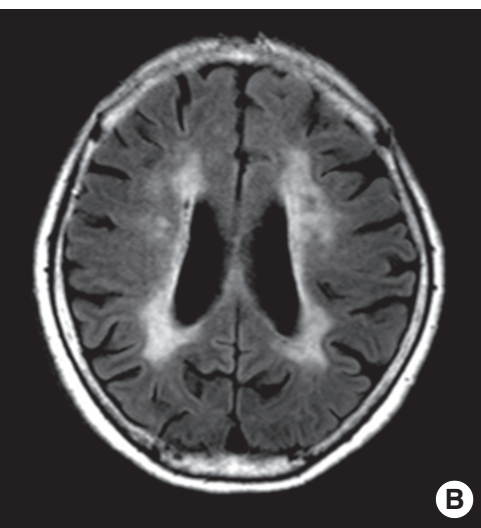

Case C

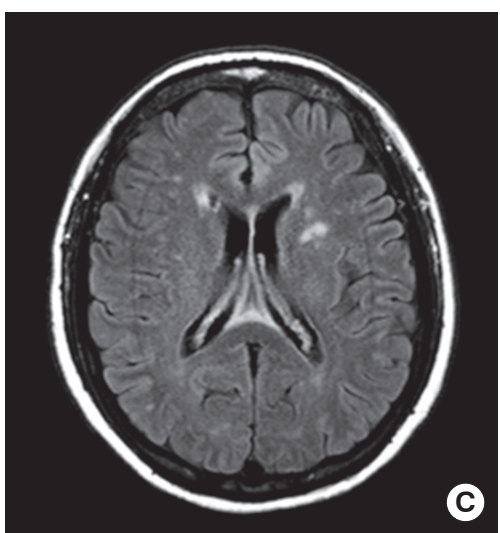

Fig. 1. Subclinical brain lesions: (A) unidentified bright objects, (B) leukoaraiosis, and (C) silent brain infarct.

Case D

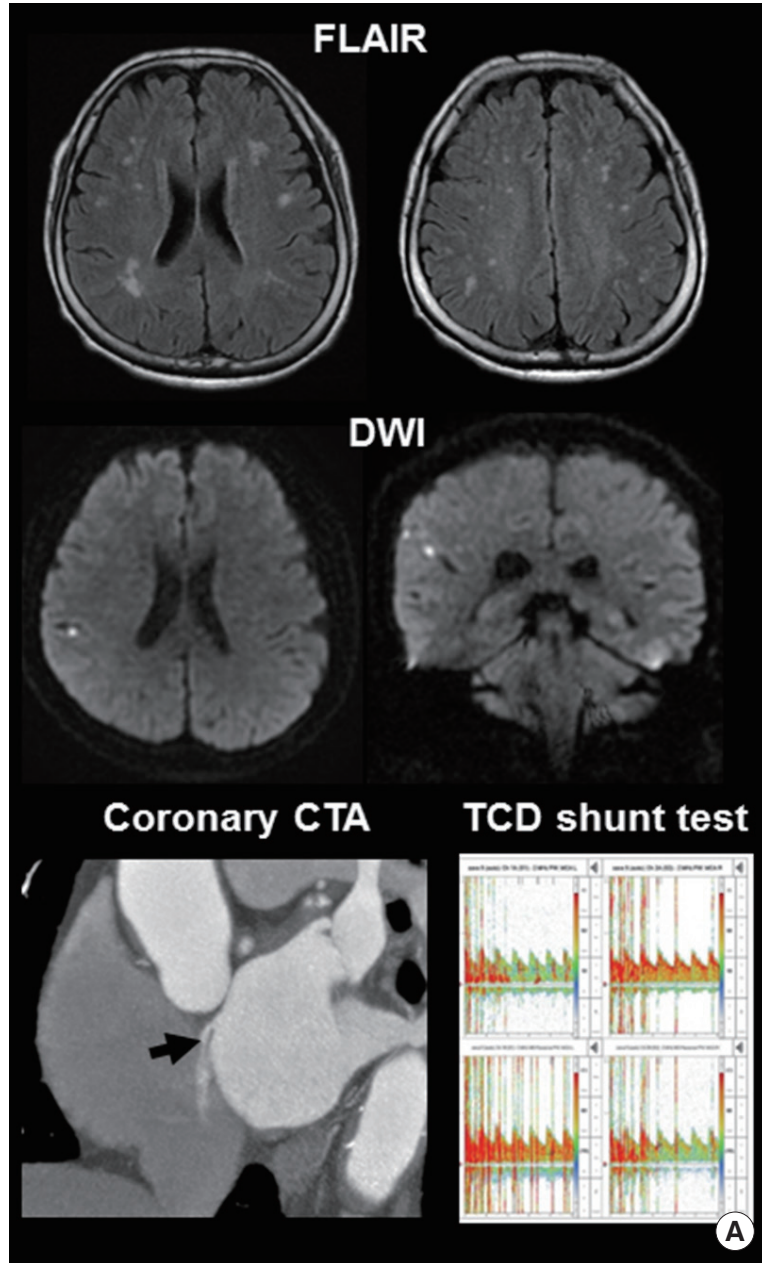

Case $\mathrm{E}$

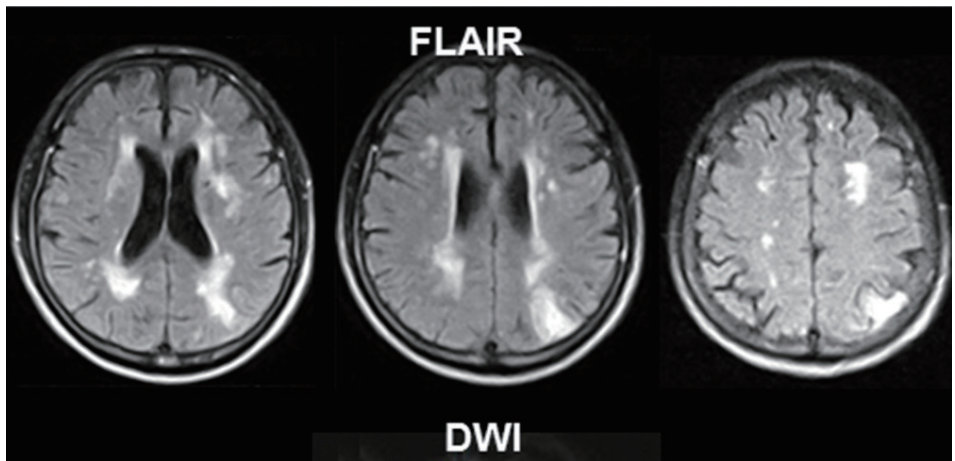

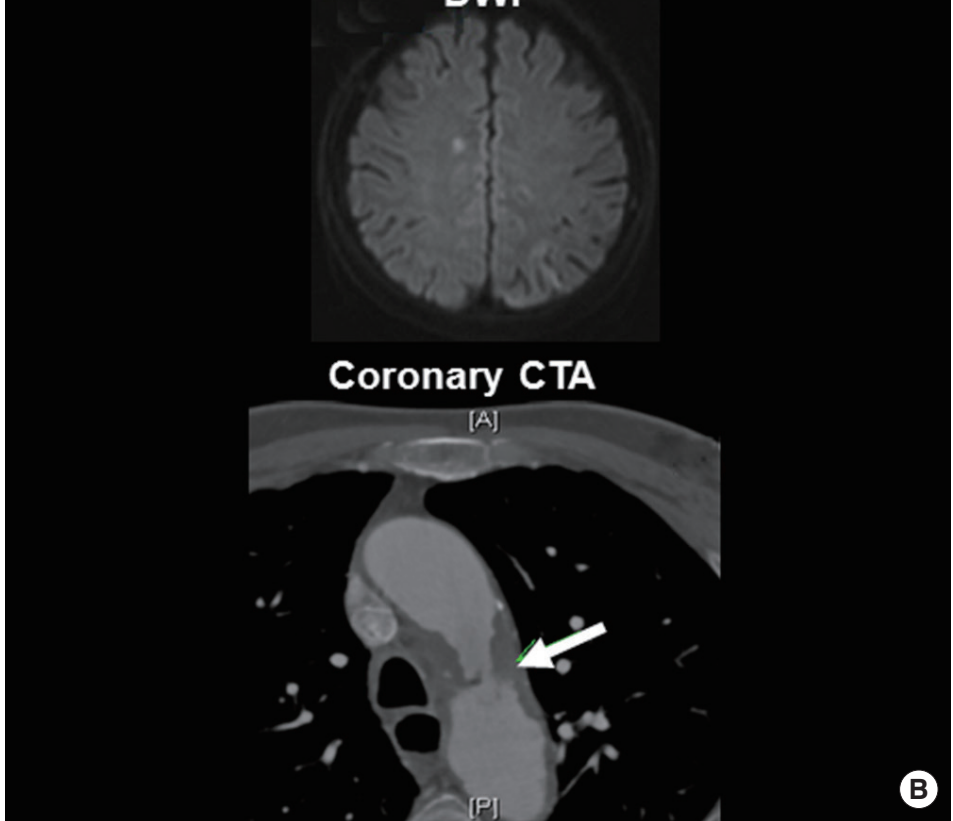

Fig. 2. Silent acute and chronic brain infarcts caused by (A) paradoxical and (B) aortogenic embolism. See details on the text. FLAIR, fluidattenuated inversion recovery; DWI, diffusion-weighted imaging; CTA, computed tomographic angiogram; TCD, transcranial Doppler. 
brain MRI for her chronic headaches. MRI showed silent small cortical infarcts on FLAIR imaging and also multiple silent but acute small cortical infarcts on diffusion-weighted imaging (DWI) (Fig. 2A). Coronary computed tomographic angiogram (CTA) showed a contrast agent jet from the left atrium to the right atrium toward the inferior vena cava, suggesting that her chronic and acute SBI was caused by paradoxical embolism (arrow in left lower image, Fig. 2A). Agitated saline transcranial Duplex monitoring diagnosed paradoxical embolism based on the intracranial detection of intravenously injected microemboli (right lower image, Fig. 2A). A 72-year-old man (case E) with a history of hypertension and current smoking underwent brain MRI for progressive visual disturbance. MRI showed multiple chronic infarcts in bilateral cortical border zones on FLAIR and acute small cortical infarcts on DWI (Fig. $2 B$ ). Coronary CTA showed protruding ulcerative plaques on the aortic arch (arrow in lower image, Fig. 2B). Antiplatelet agents and a high-intensity statin were started to prevent aortoembolism.

The cases presented above demonstrate that not all brain lesions are SBIs (case A and B), and that the causes of SBIs could be lacunar (case $\mathrm{C}$ ) or embolic (cases $\mathrm{D}$ and $\mathrm{E}$ ).

\section{DEFINITION AND EPIDEMIOLOGY OF SBI}

$\mathrm{SBI}$ is defined as imaging or neuropathological evidence of brain infarction without a history of acute neurological dysfunction attributable to the lesion [2]. MRI-defined SBIs are detected in $20 \%$ of healthy elderly people and up to $50 \%$ of patients in selected series [2,3]. Patients with acute stroke or coronary artery disease show a high prevalence of SBI $[2,3]$.

The sizes of SBIs are variable, but they are usually smaller than clinical stroke infarcts. Beside the size, other factors may also determine the presence or absence of clinical symptoms at the time of brain infarct, including location of the infarct within the primary motor, sensory, or language areas of the brain, the severity of ischemic injury, and the presence of preexisting brain injury. For example, cortical lesions in posterior border zones may be asymptomatic, whereas smaller cortical lesions in the hand knob area of the motor cortex could cause severe hand weakness. Likewise, a small new SBI could be symptomatic if patients had preexisting leukoaraiosis or stroke.

The MRI diagnostic criteria for SBI remain to be settled. A review of MRI diagnostic criteria for SBI found substantial variability among 45 studies of this issue [4]. According to that review, the majority used a size threshold of $\geq 3 \mathrm{~mm}$, with excellent reliability. SBIs should be differentiated from white matter hyperintensities or leukoaraiosis, which are often considered to be secondary to ischemia, but may also reflect non-ischemic pathology, including edema, inflammation, demyelination, and gliosis [2]. Proposed criteria for SBI include the presence of three types of lesions on MRI: (1) a focus demonstrating restricted diffusion (DWI-positive), (2) a cavitary lesion, or (3) T2 hyperintense/T1 hypointense lesions [5]. Unlike SBIs, white matter hyperintensities show isointensity on T1-weighted images. Recent American Stroke/Heart Association guidelines suggest minimum MRI acquisition stan-

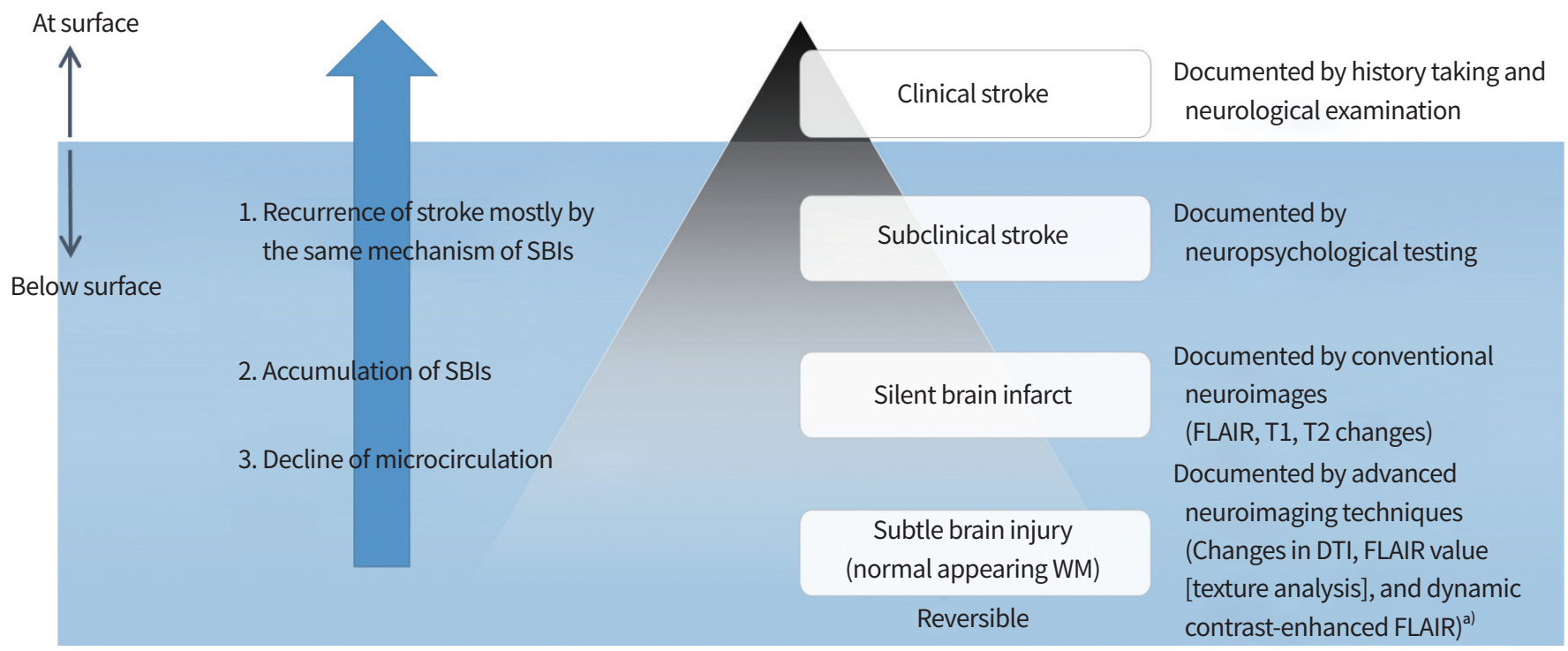

Fig. 3. Schematic overview of silent to clinically apparent cerebral infarcts. SBI, silent brain infarct; WM, white matter; FLAIR, fluidattenuated inversion recovery; DTI, diffusion tensor image. ${ }^{\text {a) }}$ To demonstrate the blood-brain barrier disruption. 
dards for the diagnosis of SBIs [6]. However, recent neuropathological studies and serial MRI studies showed that SBIs could underlie the development of leukoaraiosis and white matter hyperintensity, suggesting that clear differentiation of white matter changes from SBI may not be possible [7-9]. Fig. 3 shows the overview of silent to clinical apparent cerebral infarcts. Clinical stroke is only the emerging part of the iceberg. $\mathrm{SBI}$ and more recently introduced the normal appearing white matter are the hidden part of the iceberg. The normal appearing white matter is associated with subtle, reversible brain injury but may progress to severe white matter hyperintensity and silent infarcts [10]. Advanced MRI techniques, such as diffusion tensor image or texture analysis, are required to detect this type of brain damage $[11,12]$.

\section{CLINICAL IMPACT OF SBI}

The presence of SBI increases the risk of subsequent stroke and dementia. Large observational population-based studies and meta-analyses showed that SBI is associated with an approximately two-fold increase in risk of subsequent stroke and dementia [2,13-15]. One meta-analysis showed that the prevalence of SBI in patients with stroke who were followed up for stroke recurrence was $27.5 \%$, and that the presence of $\mathrm{SBI}$ increased the risk of stroke recurrence (adjusted hazard ratio, 2.00; $P=0.03$ ) [13]. Similarly, silent DWI lesions were observed in $3.8 \%$ to $5 \%$ of elderly individuals without stroke, and such DWI lesions were estimated to be associated with an annual incidence of hundreds of new SBI [16]. These findings suggest that $\mathrm{SBI}$ is common in both stroke-free and stroke patients, and that SBIs have similar impact on future stroke occurrence in both groups. A prospective serial follow-up MRI study showed that new SBIs after stroke were found in $24.4 \%$ of patients on day $5 \mathrm{MRI}$ and in an additional $7.4 \%$ on day $30 \mathrm{MRI}$ [17]. For these reasons, recent clinical trials of antiplatelets (TOSS-2 trial, clinicaltrials.gov identifier NCT00130039) [18], statins (EUREKA trial, NCT01364220) [19], and neuroprotective agents (ESCAPE-NA1 trial, NCT02930018) have increasingly used the SBIs detected by DWI as a surrogate marker in stroke patients.

More studies are needed to test whether the distribution and burden of SBIs influence the development of cognitive or neuropsychiatric disorders. A population-based follow-up study showed that patients with SBIs decline faster in cognition and have an increased risk of dementia compared to those free of SBI [20]. In that study, SBIs located in subcortical areas contributed more than those located in cortical and cerebellar areas, suggesting that SBIs of small vessel disease origin contribute more to the development of dementia than SBls of embolic origin.

Careful examination for covert symptoms and subtle but clinically detectable neurological abnormalities is needed in patients presumed to have SBIs. Although SBIs, by definition, lack clinically overt symptoms, they are associated with subtle deficits in brain functions that commonly go unnoticed. Whether SBIs are truly asymptomatic is still a subject of debate [21]. Elderly individuals and their family often are not aware that the symptoms they have experienced were caused by a cerebral infarct, whereas careful history taking and examination including neurological examination and neuropsychological testing may reveal these subclinical deficits. Recent clinical studies showed that covert neurological symptoms (unrecognized or unreported but clinically detectable stroke-like symptoms) were associated with the prevalence of SBIs and also with cognitive and functional decline in midlife adults or the elderly [22-24].

\section{ETIOPATHOLOGIC SUBTYPES OF SBI}

To identify the mechanism of stroke and prevent recurrent vascular events, stroke subtyping using a validated etiological classification system (e.g., TOAST and A-S-C-O) is important in patients with acute ischemic cerebrovascular disease [25-28]. Specifically, patients with cortical infarcts due to atheroembolism often have recurrent stroke with cortical infarcts within the same arterial system, whereas patients with deep infarcts caused by branch occlusive disease develop recurrent branch occlusion. Similarly, patients with small artery occlusion show recurring lacunar stroke, whereas those with cardioembolic stroke show recurrence manifesting as large cortical infarcts, regardless of the presence of asymptomatic stenosis in the cerebrovascular system.

Such stroke subtyping could also be applied in patients with SBIs. Most SBIs are lacunes, for which hypertensive small vessel disease is thought to be the main cause. In population studies, $>90 \%$ of SBIs correspond to lacunar infarcts, defined as small subcortical infarcts of 3 to $15 \mathrm{~mm}$, whereas the remaining $10 \%$ correspond to larger subcortical infarcts or cortical infarcts [29]. Age and hypertension are the most widely accepted risk factors for SBI [3]. However, the importance of SBls caused by small embolisms from the heart or aorta is increasingly recognized. SBIs have been associated with various cardiac diseases (e.g., atrial fibrillation, left ventricular thrombus, cardiomyopathy, and patent foramen 
ovale [PFO]) and procedures (e.g., transcatheter aortic valve implantation, coronary artery bypass graft surgery, and left heart catheterization) [21]. Because cardioembolic stroke is one of the common subtypes of stroke, cardioembolism could be an important mechanism of SBI. In the Framingham Offspring study, a population-based study, 2,040 patients free of clinical stroke underwent volumetric brain MRI [30]. The risk factors previously associated with clinical stroke were also found to be associated with SBIs, and included hypertension, carotid stenosis, and atrial fibrillation, suggesting a role of arterial and cardiogenic embolism in the development of SBIs. Among stroke-free patients, those with atrial fibrillation had a higher prevalence and number of SBIs and worse cognitive performance than those with sinus rhythm [31]. In patients with first-ever stroke, the presence of cardioembolic sources (e.g., atrial fibrillation) was associated with unrecognized cerebral infarcts involving multiple territories on FLAIR [32]. In our study of 1,103 healthy individual who underwent MRI, $31 \%$ had one or more superficially located SBIs, and the prevalence of right-to-left shunt was increased in those patients [33]. However, stroke subtypes or etiologic mechanisms of SBI are not well studied, and it remains to be determined which etiologic mechanisms are associated with SBI more than with clinical stroke. More studies are needed in patients with SBI to see if specific FLAIR patterns associated with SBI may guide appropriate selection of patients for specific diagnostic tests. Recent American Stroke/Heart Association guidelines recommended investigations for patients with SBIs, including carotid imaging when SBI appear in the carotid territory, echocardiography when there is an embolic-appearing pattern, and vascular imaging when large $(>1.0 \mathrm{~cm})$ silent hemorrhage is observed [6].

There have been no randomized controlled trials targeted specifically to prevention of stroke in participants with SBIs. A longitudinal study suggested a yearly incidence of $3 \%$ to $4 \%$ among the elderly (aged $>60$ years) [34]. The annual incidence of new SBIs may differ depending on the definition of an $\mathrm{SBI}$ as well as the age of the population. Further prospective studies are needed to evaluate changes in the number of SBIs in serial MRI studies, and changes in subtle brain functions, including cognitive impairments, depending on the distribution of SBIs and their underlying pathological mechanisms. Similarly, the prevention of paradoxical embolism by PFO closure and aortogenic embolism with aggressive medical management (including statins) deserves further study. For example, SBIs could be a surrogate marker of the possibility of preventing strokes by control of hypertension, the most widely accepted risk factor for SBI. A recent randomized controlled trial of PFO closure used SBIs on follow-up images as a surrogate marker to test whether PFO closure in patients with cryptogenic embolic stroke prevents recurrent stroke [35].

Should we classify SBIs as prior strokes? Or should we treat patients based on the presumed mechanism causing SBIs? Further studies are needed to justify treating SBIs as prior strokes and treating individuals for secondary prevention as aggressively as patients with clearly symptomatic infarcts. It is not clear whether anticoagulation is indicated in subjects with atrial fibrillation and SBIs and whether asymptomatic subjects with carotid stenosis and SBIs should be considered to have a higher benefit from carotid intervention than those without SBIs [2]. Recent American Stroke/Heart Association guidelines recommend that primary stroke prevention is indicated in patients with SBIs, white matter hyperintensities, or microbleeds [6]. These guidelines state that the effectiveness of aspirin to prevent stroke has not been studied, and the role of SBIs in deciding on anticoagulation for atrial fibrillation and revascularization for carotid stenosis have not been studied in randomized controlled trials.

\section{RECENT ADVANCES: MACHINE LEARNING TECHNIQUES}

Machine learning is a branch of artificial intelligence. Applications of artificial intelligence to stroke are increasing, and include diagnosis of acute ischemic stroke [36], prediction of stroke [37], predicting outcome after endovascular therapy [38], and pervasive health monitoring using smart monitoring devices [39]. Several studies of machine learning techniques to measure the severity of subclinical white matter changes have recently been reported [40], such as automated white matter segmentation algorithms to measure the severity of white matter hyperintensities in lacunar stroke $[41,42]$, support vector machine techniques to classify the burden of perivascular space in the basal ganglia region [43], and cortical and subcortical volumetric segmentation of diffusion tensor imaging feature vectors [44].

Machine learning techniques can be particularly helpful in patients with SBIs, for the following reasons. First, it may not be necessary to have a human interface, because machine learning techniques use imaging features that are most predictive for SBI rather than detecting clinical features. To distinguish lacunar (microangiopathic-origin) SBI from embolic $\mathrm{SBI}$, the software does not need to consider clinical features such as stroke symptoms and vascular risk factors, and does 
PRECISION AND FUTURE MEDICINE

Silent brain infarct: an update

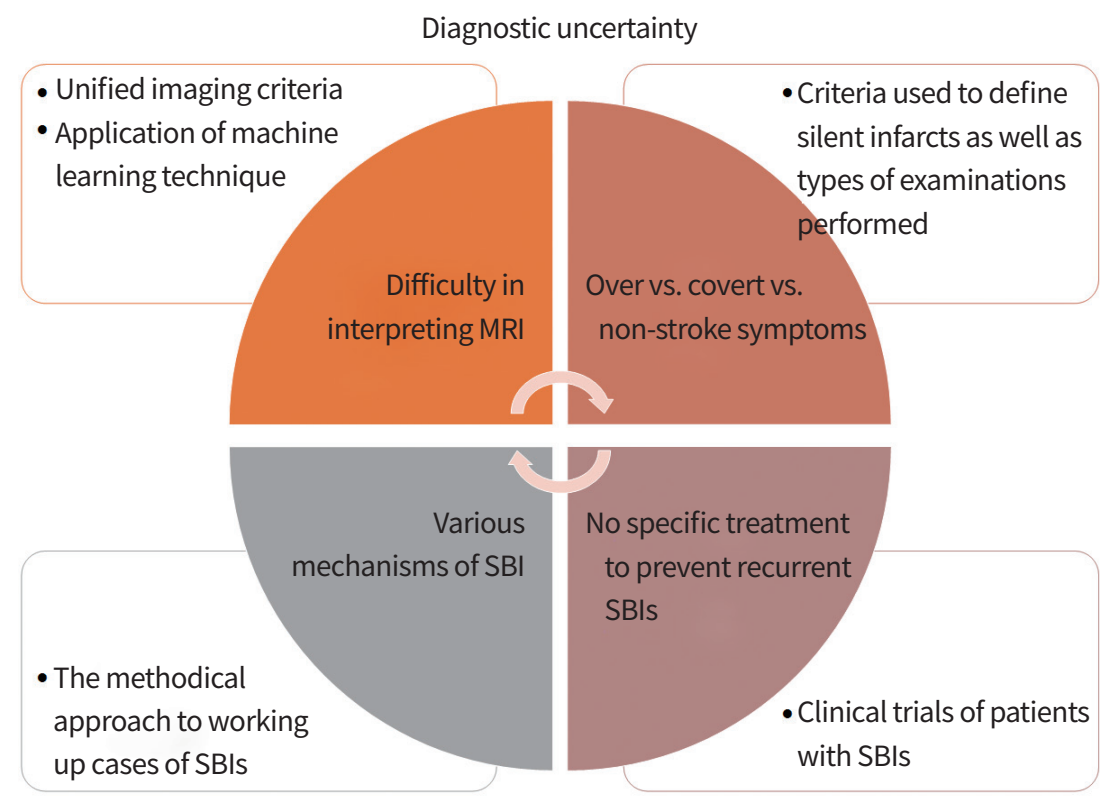

Therapeutic uncertainty

Fig. 4. Major areas of uncertainty for silent brain infarcts (SBIs) and future directions of research.

only need to classify imaging features such as the location and size of SBIs. Second, machine learning may help physician to lower the clinical burden of interpretation of MRIs in patients with possible SBIs. The number of patients with SBIS is estimated as several-fold higher than the number with clinical stroke. Third, to define SBI, not only FLAIR images, but also T1- and T2-weighted image information should be evaluated. Machine learning techniques help to merge information from various MR sequences. Lastly, as for clinical stroke $[45,46]$, patterns in the size and distribution of abnormal signals may help in identifying the underlying mechanisms of SBIs, such as lacunar or embolic pathology. We are currently developing machine learning-based automated identification and etiopathological classification systems for SBIs. However, machine learning techniques also has limitations. Their sensitivity and specificity are still suboptimal, and any of currently reported machine learning-based segmentation techniques has not been validated among different disease population and MR settings. Before validated, it should be used under the setting (target population and MR acquisition matrix) prespecified in the original study.

\section{CONCLUSIONS}

The proportion of patients diagnosed with $\mathrm{SBI}$ is high. However, SBI is an area of uncertainty (Fig. 4). Although clinicians view the prognostic significance of SBI as similar to that of clin- ical stroke, diagnostic and prognostic uncertainty has discouraged disclosure of imaging findings to patients, and therapeutic uncertainty has been common [47]. Continuous efforts are needed to improve interrater agreement in the diagnosis of $\mathrm{SBI}$ and to reduce the misclassification bias, i.e., classification of subtle ischemic changes as SBI. In addition, appropriate treatment of underlying risk factors and etiologic disease may be useful in preventing subsequent stroke and dementia. Our review of the literature, as well as cases presented within this article, suggests the need for application of appropriate workups and therapeutic agents in patients with SBI.

\section{CONFLICTS OF INTEREST}

No potential conflict of interest relevant to this article was reported.

\section{ACKNOWLEDGMENTS}

This study was supported by grants from the National Research Foundation of Korea (NRF) funded by Ministry of Science, ICT \& Future Planning (NRF-2017R1C1B2002624 and NRF-2014R1A1A1004645).

\section{ORCID}

Oh Young Bang https://orcid.org/0000-0002-7962-8751 


\section{REFERENCES}

1. O'Sullivan M. Leukoaraiosis. Pract Neurol 2008;8:26-38.

2. Sacco RL, Kasner SE, Broderick JP, Caplan LR, Connors JJ, Culebras A, et al. An updated definition of stroke for the 21st century: a statement for healthcare professionals from the American Heart Association/American Stroke Association. Stroke 2013;44:2064-89.

3. Vermeer SE, Longstreth WT Jr, Koudstaal PJ. Silent brain infarcts: a systematic review. Lancet Neurol 2007;6:611-9.

4. Zhu YC, Dufouil C, Tzourio C, Chabriat H. Silent brain infarcts: a review of MRI diagnostic criteria. Stroke 2011;42:1140-5.

5. Fanning JP, Wesley AJ, Wong AA, Fraser JF. Emerging spectra of silent brain infarction. Stroke 2014;45:3461-71.

6. Smith EE, Saposnik G, Biessels GJ, Doubal FN, Fornage M, Gorelick PB, et al. Prevention of stroke in patients with silent cerebrovascular disease: a scientific statement for healthcare professionals from the American Heart Association/American Stroke Association. Stroke 2017;48:e44-71.

7. Gouw AA, van der Flier WM, Fazekas F, van Straaten EC, Pantoni L, Poggesi A, et al. Progression of white matter hyperintensities and incidence of new lacunes over a 3-year period: the leukoaraiosis and disability study. Stroke 2008;39:141420.

8. Smith EE. Leukoaraiosis and stroke. Stroke 2010;41(10 Suppl):S139-43.

9. Conklin J, Silver FL, Mikulis DJ, Mandell DM. Are acute infarcts the cause of leukoaraiosis? Brain mapping for 16 consecutive weeks. Ann Neurol 2014;76:899-904.

10. van Leijsen EMC, Bergkamp MI, van Uden IWM, Ghafoorian M, van der Holst HM, Norris DG, et al. Progression of white matter hyperintensities preceded by heterogeneous decline of microstructural integrity. Stroke 2018;49:1386-93.

11. Gons RA, de Laat KF, van Norden AG, van Oudheusden LJ, van Uden IW, Norris DG, et al. Hypertension and cerebral diffusion tensor imaging in small vessel disease. Stroke 2010;41:2801-6.

12. Tozer DJ, Zeestraten E, Lawrence AJ, Barrick TR, Markus HS. Texture analysis of T1-weighted and fluid-attenuated inversion recovery images detects abnormalities that correlate with cognitive decline in small vessel disease. Stroke 2018;49:1656-61.

13. Gupta A, Giambrone AE, Gialdini G, Finn C, Delgado D, Gutierrez J, et al. Silent brain infarction and risk of future stroke: a systematic review and meta-analysis. Stroke 2016; 47:719-25.

14. Vermeer SE, Hollander M, van Dijk EJ, Hofman A, Koud- staal PJ, Breteler MM, et al. Silent brain infarcts and white matter lesions increase stroke risk in the general population: the Rotterdam Scan Study. Stroke 2003;34:1126-9.

15. Bernick C, Kuller L, Dulberg C, Longstreth WT Jr, Manolio T, Beauchamp N, et al. Silent MRI infarcts and the risk of future stroke: the cardiovascular health study. Neurology 2001;57:1222-9.

16. Auriel E, Westover MB, Bianchi MT, Reijmer Y, Martinez-Ramirez S, Ni J, et al. Estimating total cerebral microinfarct burden from diffusion-weighted imaging. Stroke 2015;46:2129-35.

17. Kang DW, Han MK, Kim HJ, Sohn H, Kim BJ, Kwon SU, et al. Silent new ischemic lesions after index stroke and the risk of future clinical recurrent stroke. Neurology 2016;86:27785.

18. Kwon SU, Hong KS, Kang DW, Park JM, Lee JH, Cho YJ, et al. Efficacy and safety of combination antiplatelet therapies in patients with symptomatic intracranial atherosclerotic stenosis. Stroke 2011;42:2883-90.

19. Heo JH, Song D, Nam HS, Kim EY, Kim YD, Lee KY, et al. Effect and safety of rosuvastatin in acute ischemic stroke. J Stroke 2016;18:87-95.

20. Sigurdsson S, Aspelund T, Kjartansson O, Gudmundsson EF, Jonsdottir MK, Eiriksdottir G, et al. Incidence of brain infarcts, cognitive change, and risk of dementia in the general population: the ages-Reykjavik study (age gene/ environment susceptibility-Reykjavik study). Stroke 2017;48:2353-60.

21. Hassell ME, Nijveldt R, Roos YB, Majoie CB, Hamon M, Piek $J J$, et al. Silent cerebral infarcts associated with cardiac disease and procedures. Nat Rev Cardiol 2013;10:696-706.

22. Windham BG, Griswold ME, Shibata D, Penman A, Catellier DJ, Mosley TH Jr. Covert neurological symptoms associated with silent infarcts from midlife to older age: the Atherosclerosis Risk in Communities study. Stroke 2012;43:121823.

23. Inzitari M, Pozzi C, Ferrucci L, Chiarantini D, Rinaldi LA, Baccini M, et al. Subtle neurological abnormalities as risk factors for cognitive and functional decline, cerebrovascular events, and mortality in older community-dwelling adults. Arch Intern Med 2008;168:1270-6.

24. Smith EE, O'Donnell M, Dagenais G, Lear SA, Wielgosz A, Sharma M, et al. Early cerebral small vessel disease and brain volume, cognition, and gait. Ann Neurol 2015;77:251-61.

25. Shin DH, Lee PH, Bang OY. Mechanisms of recurrence in subtypes of ischemic stroke: a hospital-based follow-up study. Arch Neurol 2005;62:1232-7. 


\section{PRECISION AND FUTURE MIEDICINE}

Silent brain infarct: an update

26. Toni D, Di Angelantonio E, Di Mascio MT, Vinisko R, Bath PM; PRoFESS Study Group. Types of stroke recurrence in patients with ischemic stroke: a substudy from the PRoFESS trial. Int J Stroke 2014;9:873-8.

27. Ay H, Furie KL, Singhal A, Smith WS, Sorensen AG, Koroshetz WJ. An evidence-based causative classification system for acute ischemic stroke. Ann Neurol 2005;58:688-97.

28. Amarenco P, Bogousslavsky J, Caplan LR, Donnan GA, Hennerici MG. New approach to stroke subtyping: the A-S-C-O (phenotypic) classification of stroke. Cerebrovasc Dis 2009; 27:502-8.

29. Vermeer SE, Koudstaal PJ, Oudkerk M, Hofman A, Breteler MM. Prevalence and risk factors of silent brain infarcts in the population-based Rotterdam Scan Study. Stroke 2002;33:21-5.

30. Das RR, Seshadri S, Beiser AS, Kelly-Hayes M, Au R, Himali $\mathrm{JJ}$, et al. Prevalence and correlates of silent cerebral infarcts in the Framingham offspring study. Stroke 2008;39:2929-35.

31. Gaita F, Corsinovi L, Anselmino M, Raimondo C, Pianelli M, Toso $\mathrm{E}$, et al. Prevalence of silent cerebral ischemia in paroxysmal and persistent atrial fibrillation and correlation with cognitive function. J Am Coll Cardiol 2013;62:1990-7.

32. Cho AH, Kwon SU, Kim TW, Lee SJ, Shon YM, Kim BS, et al. High prevalence of unrecognized cerebral infarcts in first-ever stroke patients with cardioembolic sources. Eur J Neurol 2009;16:838-42.

33. Kim SJ, Shin HY, Ha YS, Kim JW, Kang KW, Na DL, et al. Paradoxical embolism as a cause of silent brain infarctions in healthy subjects: the ICONS study (Identification of the Cause of Silent Cerebral Infarction in Healthy Subjects). Eur J Neurol 2013;20:353-60.

34. Fanning JP, Wong AA, Fraser JF. The epidemiology of silent brain infarction: a systematic review of population-based cohorts. BMC Med 2014;12:119.

35. Sondergaard L, Kasner SE, Rhodes JF, Andersen G, Iversen HK, Nielsen-Kudsk JE, et al. Patent foramen ovale closure or antiplatelet therapy for cryptogenic stroke. N Engl J Med 2017;377:1033-42.

36. Abedi V, Goyal N, Tsivgoulis G, Hosseinichimeh N, Hontecillas R, Bassaganya-Riera J, et al. Novel screening tool for stroke using artificial neural network. Stroke 2017;48:167881.

37. Weng SF, Reps J, Kai J, Garibaldi JM, Qureshi N. Can ma- chine-learning improve cardiovascular risk prediction using routine clinical data? PLoS One 2017;12:e0174944.

38. Asadi H, Dowling R, Yan B, Mitchell P. Machine learning for outcome prediction of acute ischemic stroke post intra-arterial therapy. PLoS One 2014;9:e88225.

39. Labovitz DL, Shafner L, Reyes Gil M, Virmani D, Hanina A. Using artificial intelligence to reduce the risk of nonadherence in patients on anticoagulation therapy. Stroke 2017;48:1416-9.

40. Cuadrado-Godia E, Dwivedi P, Sharma S, Santiago AO, Gonzalez JR, Balcells M, et al. Cerebral small vessel disease: a review focusing on pathophysiology, biomarkers, and machine learning strategies. J Stroke 2018;20:302-20.

41. Griffanti L, Zamboni G, Khan A, Li L, Bonifacio G, Sundaresan V, et al. BIANCA (Brain Intensity AbNormality Classification Algorithm): a new tool for automated segmentation of white matter hyperintensities. Neuroimage 2016;141:191205.

42. Park BY, Lee MJ, Lee SH, Cha J, Chung CS, Kim ST, et al. DEWS (DEep White matter hyperintensity Segmentation framework): a fully automated pipeline for detecting small deep white matter hyperintensities in migraineurs. Neuroimage Clin 2018;18:638-47.

43. Gonzalez-Castro V, Valdes Hernandez MDC, Chappell FM, Armitage PA, Makin S, Wardlaw JM. Reliability of an automatic classifier for brain enlarged perivascular spaces burden and comparison with human performance. Clin Sci (Lond) 2017;131:1465-81.

44. Diciotti S, Ciulli S, Ginestroni A, Salvadori E, Poggesi A, Pantoni $L$, et al. Multimodal MRI classification in vascular mild cognitive impairment. Conf Proc IEEE Eng Med Biol Soc 2015;2015:4278-81.

45. Ryoo S, Chung JW, Lee MJ, Kim SJ, Lee JS, Kim GM, et al. An approach to working up cases of embolic stroke of undetermined source. J Am Heart Assoc 2016;5:e002975.

46. Bang OY, Ovbiagele B, Kim JS. Evaluation of cryptogenic stroke with advanced diagnostic techniques. Stroke 2014; 45:1186-94.

47. Leung LY, Han PKJ, Lundquist C, Weinstein G, Thaler DE, Kent D. Clinicians' perspectives on incidentally discovered silent brain infarcts: a qualitative study. PLoS One 2018;13:e0194971. 